\section{Principe de facilitation appliqué à la restauration écologique de sites miniers dégradés : suivi des communautés ectomycorhiziennes au cours de successions végétales assistées par Acacia spirorbis}

\section{Anne Houlès}

\section{RÉSUMÉ}

Acacia spirorbis est une espèce endémique de la Nouvelle-Calédonie, capable de former des symbioses multiples avec des microorganismes du sol, bactéries fixatrices d'azote, champignons mycorhiziens arbusculaires et champignons ectomycorhiziens. Cette espèce est capable de se développer sur une très large variété de sols de $\mathrm{pH} 4$ à $\mathrm{pH} 8$, texture sableuse à argileuse, riche ou pauvre en matières organiques, etc. Afin de tester les capacités de cette espèce à initier un processus de restauration écologique des écosystèmes métallifères dégradés par l'exploitation minière, un essai en pépinière et trois essais sur le terrain ont été conduits. Ces essais ont permis de mettre en évidence la capacité d'A. spirorbis à jouer, grâce à la facilitation, un rôle de " plante nurse » pour des espèces cibles de la restauration écologique comme des espèces du genre Tristaniopsis. Ainsi, nous avons tout d'abord décrit 76 taxons moléculaires (OTUs) de champignons ectomycorhiziens dominés par des taxons proches de Tomentella et de Thelephora, associés avec $A$. spirorbis dans 12 sites répartis sur trois classes de sol : ferralitique, volcano-sédimentaire et calcaire. Nous avons ensuite constaté la capacité de ces OTUs à s'associer aux Tristaniopsis grâce à la présence initiale d'A. spirorbis. Les capacités d'A. spirorbis à faciliter l'implantation d'autres espèces en améliorant leur survie, leur croissance et en leur permettant de disposer de partenaires fongiques ectomycorhiziens diversifiés sont décrites. Ces résultats ont conduit à proposer un itinéraire technique de restauration écologique des maquis miniers dégradés, itinéraire fondé sur la facilitation entre espèces utilisant $A$. spirorbis comme plante nurse.

\section{The facilitation principle applied to ecological restoration of degraded mining sites: monitoring ectomycorrhizal communities during plant succession assisted by Acacia spirorbis}

\section{ABSTRACT}

Acacia spirorbis is a species endemic to New Caledonia, capable of forming multiple symbioses with soil microorganisms, nitrogen-fixing bacteria, arbuscular mycorrhizal fungi and ectomycorrhizal fungi. This species is capable of developing in a very wide variety of soils from $\mathrm{pH} 4$ to $\mathrm{pH}$ 8 , sandy to clayey in texture, rich or poor in organic matter, etc. In order to test the ability of this species to trigger a process of ecological restoration of metalliferous ecosystems degraded by mining activities, a nursery trial and three field trials were conducted. These trials showed how in the process of ecological restoration, $A$. spirorbis, thanks to facilitation, has the capacity to act as a nurse plant for target species like those of the genus Tristaniopsis. We first described 76 molecular taxa (OTUs) of ectomycorrhizal fungi dominated by taxa close to Tomentella and Thelephora, in association with $A$. spirorbis in 12 sites distributed over three soil classes: ferralitic, volcano-sedimentary and calcareous. We then noted the ability of these OTUs to associate with Tristaniopsis through the initial presence of $A$. spirorbis, and described the abilities of $A$. spirorbis to facilitate the establishment of other species by improving their survival and growth and by providing them with a variety of ectomycorrhizal fungal partners. Based on these results, we propose a technical scheme for the ecological restoration of degraded mining maquis, making use of ecological facilitation between species via A. spirorbis as a nurse plant.

Keywords: ectomycorrhizal, Acacia spirorbis, nurse plant, mining maquis, ecological restoration, New Caledonia.

\section{Principio de facilitación aplicado a la restauración ecológica de sitios de minería degradada: monitoreo de comunidades de ectomicorrizas durante sucesiones vegetales asistidas por Acacia spirorbis}

\section{RESUMEN}

Acacia spirorbis es una especie endémica de Nueva Caledonia, capaz de formar simbiosis múltiples con los microorganismos del suelo, bacterias fijadoras de nitrógeno, hongos micorrizas arbusculares y hongos ectomicorrizas. Esta especie es capaz de desarrollarse en una gran variedad de suelos desde $\mathrm{pH} 4$ hasta $\mathrm{pH} 8$, de textura arenosa hasta arcillosa, ricos o pobres en materia orgánica, etc. Con el fin de probar la capacidad de esta especie para iniciar un proceso de restauración ecológica de ecosistemas metalíferos degradados a través de la minería, se llevó a cabo una prueba de vivero y tres ensayos de campo. Estas pruebas permitieron poner de relieve la capacidad de $A$. spirorbis para desempeñar el papel de planta nodriza, gracias a la facilitación, hacia planta blanco como especies del género Tristaniopsis que se utilizan en el proceso de la restauración ecológica. Por lo tanto, hemos descrito por primera vez 76 taxones moleculares (OTUs) de hongos ectomicorrizas, los cuales fueron dominados por taxones Tomentella y Thelephora asociados con $A$. spirorbis en 12 sitios distribuidos en tres clases de suelos: ferralíticos, vulcano-sedimentarios y calcáreos. Luego, hemos notado la capacidad de estas OTUs de asociarse con Tristaniopsis a través de la presencia inicial de $A$. spirorbis. También hemos descrito la capacidad de $A$. spirorbis para facilitar la implantación de otras especies de plantas mediante la mejora de su supervivencia, su crecimiento y al permitirles desarrollar simbiosis con hongos ectomicorrizas. Estos resultados conducen a proponer un itinerario técnico de restauración ecológica del maquis minero degradado, basado en la facilitación entre especies que utilizan $A$. spirorbis como planta nodriza.

Palabras clave: ectomicorriza, Acacia spirorbis, planta nodriza, maquis minero, restauración ecológica, Nueva Caledonia.

Mots-clés : ectomycorhize, Acacia spirorbis, plante nurse, maquis minier, restauration écologique, NouvelleCalédonie.

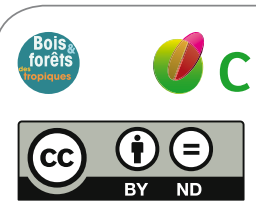

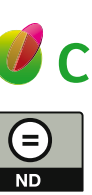
Doi : $10.19182 / \mathrm{bft} 2018.336 . \mathrm{a3} 1616$

Droit d'auteur (C) 2018, Bois et Forêts des Tropiques (C) Cirad Date de publication : $1^{\text {er }}$ avril 2018 

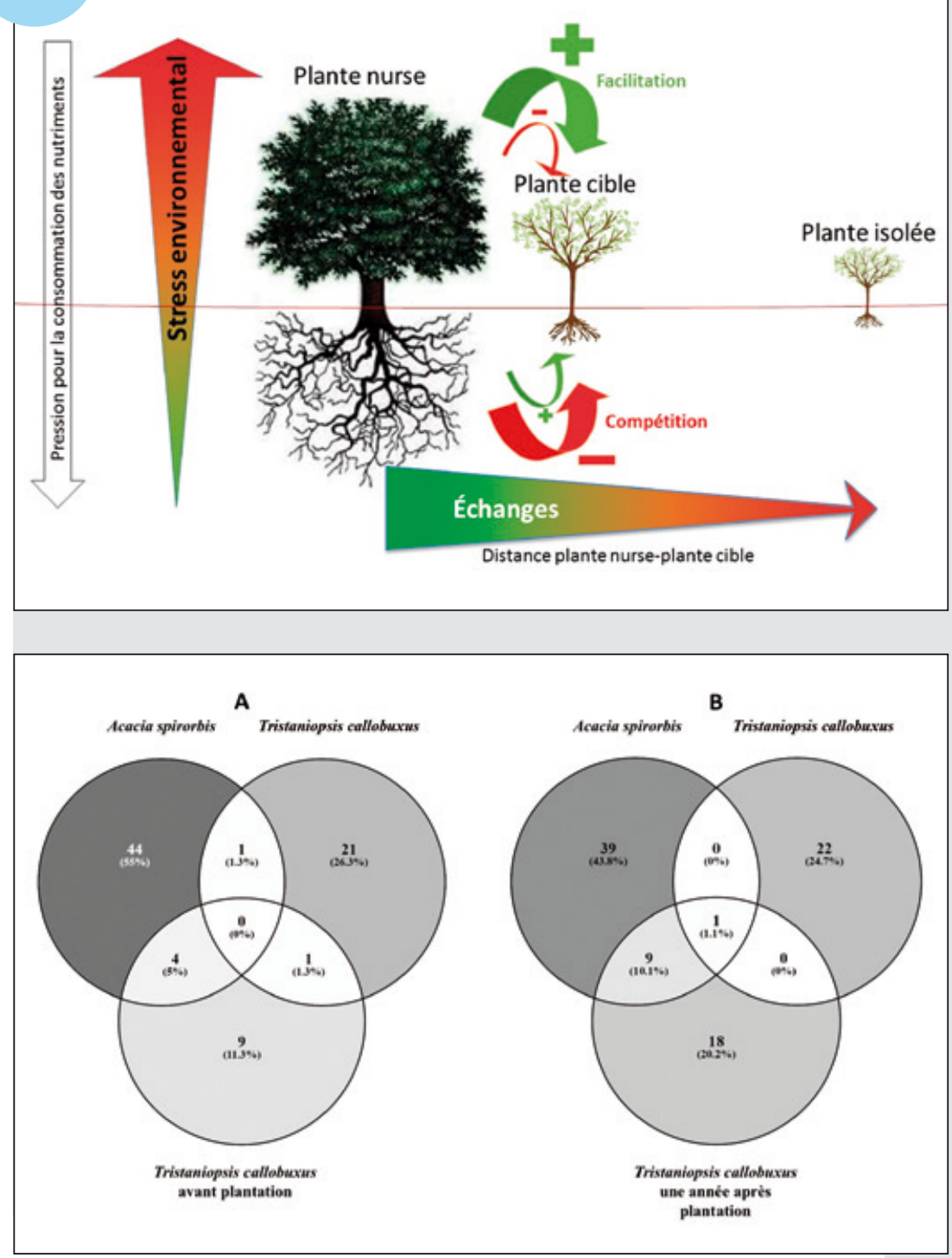

Figure 1.

Modélisation des interactions entre la plante nurse et la plante cible en fonction du stress environnemental et de la distance entre les plantes. Les interactions de compétition (flèches rouges) et de facilitation (flèches vertes) varient inversement en fonction du stress environnemental : plus le stress est élevé, plus la facilitation est importante et la compétition faible; dans les environnements peu stressants, la compétition est maximale et la facilitation très faible. Les interactions de compétition et de facilitation s'estompent avec l'augmentation de la distance entre les plantes.

Figure 1.

Modelling interactions between the nurse plant and the target plant according to environmental stress and distance between plants. Competition (red arrows) and facilitation (green arrows) interactions vary in inverse proportion to environmental stress: the higher the stress, the greater the facilitation and the weaker the competition; in low-stress environments, competition is at its maximum and facilitation very low. Competition and facilitation interactions diminish with increasing distance between plants.

Figura 1.

Modelización de las interacciones entre la planta nodriza y la planta blanco en función del estrés medioambiental y de la distancia entre las plantas. Las interacciones de competencia (flechas rojas) y de facilitación (flechas verdes) varían inversamente en función del estrés medioambiental: como más elevado es el estrés, más importante es la facilitación y más débil es la competencia. En los ambientes con poco estrés, la competencia es máxima y la facilitación muy débil. Las interacciones de competencia y de facilitación disminuyen con el aumento de la distancia entre las plantas.

Figure 2.

Illustration de l'évolution de la diversité des partenaires ectomycorhiziens grâce à la présence d'une plante nurse, Acacia spirorbis, et de la compatibilité de ses partenaires ectomycorhiziens avec une espèce cible : Tristaniopsis callobuxus Brongn. \& Gris. L'analyse par séquençage Sanger de la diversité des champignons ectomycorhiziens permet de constater après un an de plantation un accroissement significatif du nombre de taxons communs entre la plante nurse et la plante cible.

Figure 2.

Diagram illustrating how the diversity of of ectomycorrhizal partners evolves thanks to the presence of a nurse plant, Acacia spirorbis, and the compatibility of its ectomycorrhizal partners with a target species: Tristaniopsis callobuxus Brongn. \& Gris. The Sanger sequencing analysis of the diversity of ectomycorrhizal fungi shows a significant increase in the number of taxa common to the nurse plant and the target plant one year after planting.

Figura 2.

Ilustración de la evolución de la diversidad de ectomicorrizas asociadas gracias a la presencia de una planta nodriza, Acacia spirorbis, y la compatibilidad de sus ectomicorrizas asociadas con una especie blanco: Tristaniopsis callobuxus Brongn. \& Gris. El análisis de secuenciación de Sanger de la diversidad de hongos ectomicorrizas muestra un aumento significativo en el número de taxones comunes entre la planta nodriza y la planta blanco después de un año de la siembra.

Grade et diplôme : Docteur en sciences de l'Université de Montpellier. Université et école doctorale : Université de Montpellier, école doctorale GAIA (Biodiversité, Agriculture, Alimentation, Environnement, Terre, Eau).

Date de soutenance : 24 novembre 2017.

Direction et encadrement : Marc Ducousso (Chercheur CIRAD, UMR LSTM, France) et Philippe JouRAND (Ingénieur de recherche IRD, UMR LSTM, Nouvelle-Calédonie, France).

Composition du jury

Président du jury : Hamid AmIR (Professeur de l'Université de Nouvelle-Calédonie, France).

Rapporteurs : Pierre Emmanuel CourTY (Chargé de recherche INRA Dijon, France), Mélanie Roy (Maître de conférences, Université Toulouse-Paul Sabatier, France).

Examinateurs : Claude PLASSARD (Directrice de recherche, INRA Montpellier, France), Robin DuponnoIs (Directeur de recherche, IRD Montpellier, France).

Invité : Magali PATRoIS (Ingénieur environnement, Koniambo Nickel SAS, Nouvelle-Calédonie, France).

Langue de rédaction : français et anglais.

Accès au manuscrit : http://www.theses.fr/s154612

Contact : UMR LSTM, TA A-82 / J, Campus international de Baillarguet, 34398 Montpellier Cedex 5, France.

houles.anne@gmail.com ou marc.ducousso@cirad.fr https://umr-lstm.cirad.fr/

\section{Publications / Publications / Publicaciones}

Houlès A., Joussemet F., Hannibal L., Patrois M., Vedi L., Jourand P., Ducousso M., 2018. Ectomycorrhizal facilitation using Acacia spirorbis Labill. as nurse plant enable to enhance ectomycorrhizal community composition of a target species: Tristaniopsis callobuxus Brongn. \& Gris. Mycorrhiza.

Houlès A., Vincent B., David M., Ducousso M., Galiana A., Juillot F., Hannibal L., Carriconde F., Fritsch E., Jourand P., 2018. Ectomycorrhizal communities associated with the legume Acacia spirorbis growing on contrasted edaphic constraints in New Caledonia. Microbial Ecology (accepté pour publication). 